\title{
Effect of patient thickness on acute gastrointestinal toxicities following radiotherapy for prostate cancer
}

\author{
Robert Pisani, Paul Bezzina, Jose Guilherme Couto \\ Department of Radiography, Faculty of Health Sciences, University of Malta, Msida, Malta
}

\begin{abstract}
Background: There is conflicting literature regarding the effect of patient size on radiotherapy toxicities. This study aimed to determine whether there is any association between patient thickness and severity and incidence of acute GI toxicities of prostate cancer patients receiving VMAT radiotherapy. The impact of confounding factors was also examined: rectal dose, age and lymph node irradiation.

Materials and methods: This study used a non-experimental, retrospective, descriptive and cross-sectional design. All patients who complied with the inclusion criteria $(n=96)$ were included. Gl toxicity scores (baseline and last week of radiotherapy), rectal dose, lymph nodes irradiation and patient age at diagnosis were collected from the treatment file. Patient separations were measured from the CT-Simulator images. Statistical tests were performed to analyse the influence of these factors on acute $\mathrm{Gl}$ toxicities.

Results: Patient thickness was shown to have no statistically significant effect on the incidence ( $p=0.947$ for antero-posterior and $p=0.839$ for lateral thicknesses), and severity ( $p=0.986$ and 0.905 , respectively) of acute GI toxicities. Severity of GI toxicities increased following radiotherapy: the Expanded Prostate Cancer Index Composite (EPIC) score increased by 2.64 from baseline $(p<0.001)$. The confounding factors had no statistically significant effect on toxicities $(p>0.05)$.

Conclusion: As expected, most patients experienced an increase in Gl toxicity following radiotherapy. No relationship was established between patient thickness and the severity or incidence of acute Gl toxicities, adding to the existing body of knowledge. Therefore, all patients should receive adequate follow up, irrespective of size. Side-effect recording tools should be implemented systematically for continuous assessment of this relationship.
\end{abstract}

Key words: separation; thickness; patient size; gastrointestinal toxicity; prostate cancer Rep Pract Oncol Radiother 2021;26(3):352-359

\section{Introduction}

Radiotherapy is a well-established treatment for a wide range of pathologies, including cancer of the prostate [1]. Radiotherapy consists of the delivery of ionising radiation to a target in order to treat a disease, avoiding the adjacent healthy tissues. However, healthy tissues will inadvertently still be exposed, resulting in unwanted toxicities (i.e. side-effects) which are a major limitation of radiotherapy [2]. Even though these toxicities are temporary, they may result in a diminished quality of life for the patient, and increase the risk of developing late toxicities which are often permanent [3].

Prostate cancer $(\mathrm{PCa})$ is the most common malignancy in men in Malta, accounting for approximately $20 \%$ of all new male cancer diagnoses [4]. Radiotherapy is a frequently utilised treatment for

Address for correspondence: Robert Pisani, Department of Radiography, Faculty of Health Sciences, University of Malta, Msida, Malta; e-mail: robert.r.pisani.16@um.edu.mt 
PCa, which commonly causes toxicities, with as many as $90 \%$ of patients developing gastrointestinal (GI) toxicities [5].

The rationale for a relationship between patient thickness and acute GI toxicities is as follows: an increase in the patient thickness will require a higher radiation exposure in order to administer the prescribed dose to the target which is deeper in the body [6]. Therefore, the adjacent normal tissues may receive a greater radiation dose, increasing toxicities. Yet, optimisation of the treatment may allow for administration of the dose to the target while avoiding the rectum.

Although there is published literature correlating patient size with the radiotherapy toxicities internationally [7-13], there is a lack of knowledge with regards to acute GI toxicities following PCa radiotherapy specifically.

Furthermore, the research findings of such articles were conflicting $[7,9,12,13]$. The majority of studies indicated that a larger patient size tended to reduce the incidence and severity of acute GI toxicities, and was attributed to a greater distance between the rectum and target due to adipose tissue $[7,8,10,11]$. Conversely, two articles reported that larger patient sizes lead to more GI toxicities $[12,14]$. Moreover, three other articles indicated that there was no significant association between patient size and toxicities. This lack of association was attributed to the use of highly accurate radiotherapy techniques, such as volume modulated arc therapy (VMAT) and image verification, which would improve treatment accuracy, thereby limiting the exposure of the adjacent organs at risk (OARs) $[9,13,15]$.

The literature presents various methods to establish the relationship between patient size and toxicities, leading to differences in findings and hindering comparison of results. A limited number of studies made use of thickness (also referred to as "separation" in some publications) to measure patient size [16], while other studies used BMI or visceral fat $[7,8,17]$. Different toxicity grading tools were also used to measure the toxicities across the different articles [13, 18].

This study used patient thickness as the measurement of patient size, where a more significant thickness reflects a larger patient size. Antero-posterior (AP) thickness refers to the distance between the front and the back aspects of the patient's body, while the lateral thickness corresponds to the distance between the left and right sides of the patient [19]. Moreover, thickness is particularly important in radiotherapy since it directly affects dose distribution [6].

In addition, several studies focused on the impact of patient size on other aspects of radiotherapy, namely patient setup accuracy $[16,20,21]$ and radiation dosimetry [22-24], which may have an effect on radiation toxicities. Thereby, the relationship between patient size and toxicities is multifaceted.

This study aimed to determine whether there is an association between the thickness of patients receiving VMAT radiotherapy for $\mathrm{PCa}$ and the severity and incidence of acute GI radiation toxicities. The influence of pelvic lymph node (LN) irradiation, patient age and rectal dosimetry on the incidence and severity of acute GI toxicities was also evaluated. This assessment of confounding factors was not considered in previously published studies.

This study focused on PCa cases treated with VMAT, for standardisation. The scope was also limited to the effect that patient thickness has on acute GI toxicities to ensure that the aim is achievable; as such, other radiation toxicities were not investigated.

\section{Materials and methods}

\section{Population and sampling}

The target population in this study included all patients who previously underwent VMAT to the prostate in a Maltese radiotherapy centre. The focus on PCa radiotherapy cases specifically ensured that the population was homogenous, removing potential confounding factors, such as different target sizes and dose prescriptions.

Table 1 specifies the inclusion and exclusion criteria used for selecting the accessible population. Patients who did not fulfil these criteria were not included in this study. All PCa patients treated with VMAT at a Maltese radiotherapy centre who completed treatment prior to data collection were included in the study (exhaustive sampling). Furthermore, all included patients were treated with image-guided radiotherapy using cone-beam computed tomography. This allowed the sample to become more representative of the target population, leading to more accurate generalisations. 
Table 1. Selection criteria

\begin{tabular}{|l|l|}
\hline \multirow{3}{*}{ Inclusion criteria } & The patient is undergoing radiotherapy for PCa \\
& The patient has not been diagnosed with metastasis \\
The patient has received VMAT radiotherapy with radical intent & The PTV includes prostate and seminal vesicles (with or without lymph nodes) \\
The EPIC forms (baseline and last week of radiotherapy) were available & The patient has received standard hormone therapy for PCa \\
\hline \multirow{3}{*}{ Exclusion criteria } & $\begin{array}{l}\text { The patient has received surgery to the prostate (e.g. radical prostatectomy) } \\
\text { The patient has a hip prosthesis implanted } \\
\text { The PTV does not include the seminal vesicles }\end{array}$ \\
\hline
\end{tabular}

PCa — prostate cancer; VMAT — volume modulated arc therapy; PTV — planning target volume; EPIC — Expanded Prostate Cancer Index Composite

Patients who had previously undergone a transurethral resection of the prostate were still included, since no dosimetric alterations are performed in such cases [25]. The inclusion of only patients with seminal vesicle irradiation in addition to the prostate allowed for similar treatment volumes between patients. At a Maltese radiotherapy centre where this study took place, all radical prostate cancer patients receive the same dose prescription, which is: $60 \mathrm{~Gy}$ in 20 fractions over 4 weeks. Therefore, all included patients received the same total doses.

\section{Data collection tools}

The data was collected retrospectively by two intermediaries, who were experienced radiographers working at a Maltese radiotherapy centre. This was done to ensure the anonymity of patients.

The Expanded Prostate Cancer Index (EPIC) tool was used to collect patient data on GI toxicity incidence and severity. This tool allows for an assessment of OAR function and the degree of inconvenience for the patient from any toxicities being experienced [26]. The EPIC tool was an established and validated instrument tested with a large randomly-selected cohort of patients, whose characteristics and demographics were internationally representative [27].

At a Maltese radiotherapy centre, EPIC is performed several times through radiotherapy treatments. For this study the EPIC scores before starting radiotherapy (baseline) and during the last week of radiotherapy were collected (follow-up). This allowed for an assessment of the contribution of radiotherapy to the toxicity score following treatment.

A data record sheet was used to collect the following data from each patient: Patient thickness (AP and lateral), EPIC score (baseline and follow-up), rectal dose (D80\%), LN irradiation and age. The validity and reliability of the data record sheet were tested, and it was found to be both valid and reliable. Validity was tested using the Content Validity Index for Items from the two experienced radiographers. The Item Content Validity Index (ICVI) was 1.00, demonstrating a suitable level of validity for the data record sheet. Moreover, reliability was established by having the two intermediaries separately collect the data from the same five patients who had satisfied the inclusion and exclusion criteria and document it on separate data record sheets accordingly. The intraclass correlation coefficient (ICC) was then calculated to be 1.00 , indicating excellent reliability.

In the context of this study, "incidence" of acute GI toxicities occured when the EPIC total score was greater in the last week of radiotherapy when compared with the baseline. In addition, "severity" was the difference in EPIC total scores between the last week of treatment and baseline, with larger changes in scores being indicative of greater severity.

The EPIC scores, patient age at diagnosis, pelvic LN irradiation status and rectum dose-volume parameters were obtained through the patients' medical files. The baseline EPIC scores were selected as at a date before starting PCa radiotherapy, whereas the follow-up EPIC scores were required to have been completed during the last week of radiotherapy or the week following completion.

The patient AP and lateral thicknesses were measured through the Computed Tomography-Simulator (CT-Sim) images. Both AP and lateral thickness were measured at the level of the prostate base, since this is close to the centre of the radiation beams where the thickness will influence dose distribution the most [1]. To ensure reproducible measurements clear instructions for the measurement of the thickness were provided to the intermediar- 
Table 2. Statistical tests used for data analysis

\begin{tabular}{|l|l|}
\hline Statistical test & Use \\
\hline Shapiro-Wilk test & Assessment of normality of data distribution \\
\hline Spearman's rank correlation coefficient & $\begin{array}{l}\text { Correlation between patient thickness and Gl toxicity severity } \\
\text { Correlation between rectal dose-volume parameter and severity of Gl toxicities }\end{array}$ \\
\hline Kruskal-Wallis test & $\begin{array}{l}\text { Comparison of Gl toxicity severity between patients with and without nodal irradiation } \\
\text { Comparison of Gl toxicity severity between age-groups } \\
\text { Comparison of thickness between patients who have and have not develop GI toxicities } \\
\text { Comparison of rectal dose between patients who have and have not develop Gl toxicities }\end{array}$ \\
\hline Chi-square test & $\begin{array}{l}\text { Comparison of incidence between patients with and without nodal irradiation } \\
\text { Comparison of incidence between age-groups }\end{array}$ \\
\hline Wilcoxon signed-rank test & Incidence of toxicities between baseline and follow-up \\
\hline
\end{tabular}

GI — gastrointestinal

ies: the AP thickness was measured at the patient's midline, while the lateral thickness was measured at the antero-posterior midpoint of the prostate gland, both measurements were performed at the base of the prostate gland. The same procedure was repeated for all patients. When multiple CT-Sim scans were available, the scan which was used for treatment planning was utilised. Rectal dose-volume parameters were obtained through the printed treatment plan. The parameter recorded was the dose to $80 \%$ of the rectum volume.

\section{Statistical analysis}

IBM SPSS version 26 was used to analyse the data. Several non-parametric statistical tests were used for data analysis, summarised in Table 2. Significance was taken when the p-value was lower than the 0.05 level of significance [28].

\section{Ethical considerations}

Prior to the study, ethical clearance was obtained from the University Research and Ethics Committee at the University of Malta. Confidentiality was ensured since the identity of patients was not disclosed in the research data. Each patient was as- signed a code by the intermediaries, which was known to them only.

\section{Results}

A total of 120 PCa patients were treated since the introduction of VMAT to treat this pathology, with the EPIC forms also being available. An exhaustive sampling technique was used, and 96 patients were included, following the selection criteria (Tab. 1).

The majority of patients were within the 71 to 75 age-group (38.5\%), which was expected since the mean age at diagnosis of $\mathrm{PCa}$ is 72 years [29]. The mean AP thickness was 22.87 centimetres while mean lateral thickness was 36.60 centimetres, with a standard deviation of 2.64 and 2.45 , respectively.

The mean dose to $80 \%$ of the rectum was $42.26 \mathrm{~Gy}$ when an incidence of toxicities was reported and 44.04Gy without incidence. A total of 42 patients reported an incidence of GI toxicities, while 54 patients reported no increase, meaning the ratio of 7:9. The small number of patients with LN irradiation $(6.3 \%)$ may limit the generalisation of results regarding $\mathrm{LN}$, since such a small sample may not be representative (Tab. 3).

Table 3. Demographics

\begin{tabular}{|c|c|c|c|}
\hline & & Frequency & Percentage \\
\hline \multirow{4}{*}{ Patient age-groups } & 65 years or less & 21 & $21.9 \%$ \\
\hline & $66-70$ years & 21 & $21.9 \%$ \\
\hline & $71-75$ years & 37 & $38.5 \%$ \\
\hline & 76 years or more & 17 & $17.7 \%$ \\
\hline \multirow{2}{*}{ Pelvic lymph nodes irradiation status } & Yes (included) & 6 & $6.3 \%$ \\
\hline & No (not included) & 90 & $93.8 \%$ \\
\hline
\end{tabular}



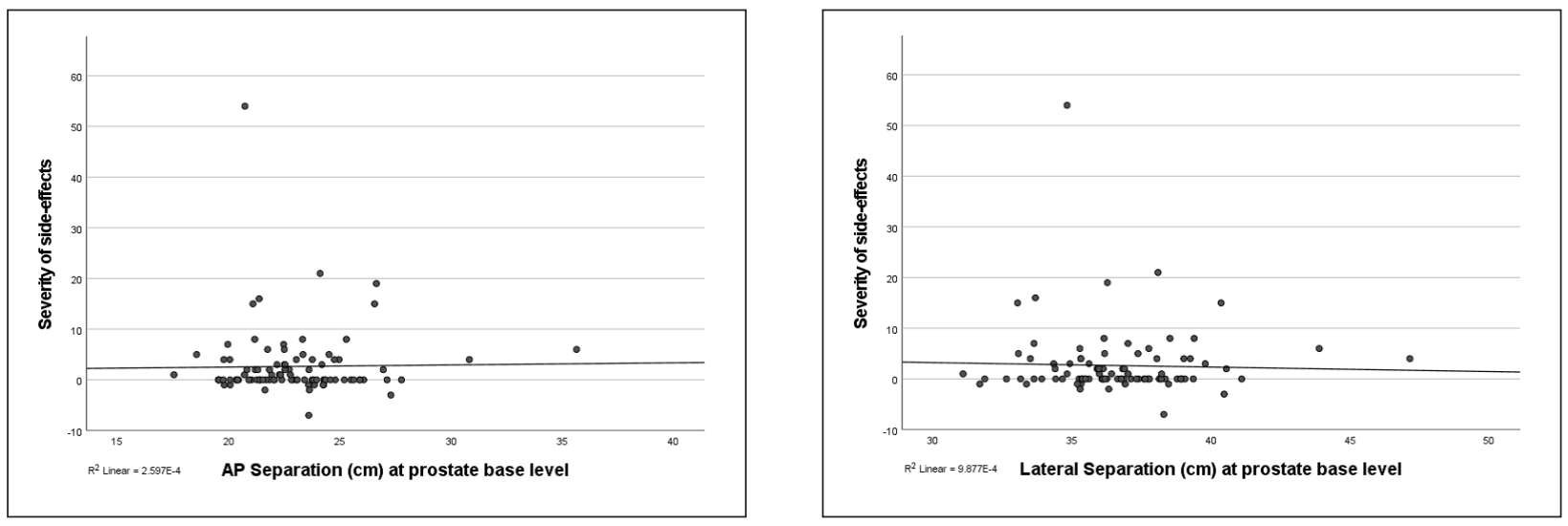

Figure 1. Scatter graph correlating severity with antero-posterior (AP) and lateral thicknesses

Most patients showed few GI toxicities throughout the treatment period, yet, a significant increase ( $\mathrm{p} \leq 0.001$ ) was observed between the baseline (mean EPIC score $=0.36$ ) and after radiotherapy (mean EPIC score $=3.00$ ). The relationship between the incidence and the severity of acute GI toxicities with thickness, rectal dose, LN irradiation and patient age are explored below.

\section{Relationship between thickness and Gl toxicities}

The Spearman correlation coefficients relating toxicity severity to AP and lateral thickness were both close to zero (Rs $=0.002$ for AP thickness and Rs $=0.012$ for lateral thickness), indicating a very weak relationship. Moreover, the p-values for AP and lateral thickness ( 0.986 and 0.905 , respectively) show that the correlation was not statistically significant (Fig. 1).

There was a non-significant difference $(\mathrm{p}=0.947)$ between the AP thickness of patients who showed an incidence of GI toxicities (AP thickness $=23.0$ $\mathrm{cm}$ ) in comparison with those who did not develop GI toxicities (AP thickness $=22.8 \mathrm{~cm}$ ). In addition, the mean lateral thickness also marginally differed between incidence groups, with a mean lateral thickness of $36.73 \mathrm{~cm}$ for the group with an incidence of toxicities and of $36.50 \mathrm{~cm}$ for the group without an incidence of toxicities.

\section{Correlation between rectal dose and GI toxicities}

The Spearman correlation coefficient showed a non-significant $(\mathrm{p}=0.535)$ and very weak correlation between rectal dose and toxicity severity
$(\mathrm{Rs}=-0.064)$. Moreover, upon analysis, it was shown that there was no significant difference $(\mathrm{p}=0.404)$ in the mean dose to $80 \%$ of the rectum between the patient cohort with (mean D80\% $=42.26 \mathrm{~Gy}$ ) and without (mean D80\% = 44.04 Gy) incidence of GI toxicities.

\section{Relationship between LN irradiation and $\mathrm{Gl}$ toxicities}

The Kruskal-Wallis test indicated that the mean severity of toxicities for patients who did not receive LN irradiation was only marginally higher than the mean severity with LN irradiation $(\mathrm{p}=0.880)$. The Chi-Square test showed that the incidence of GI toxicities was similar between patients with pelvic $\mathrm{LN}$ irradiation and those without $(\mathrm{p}=0.595)$. Thereby, no significant association was found between LN irradiation and GI toxicities. The lack of statistical significance may be due to the small size of the group with LN irradiation $(n=6)$.

\section{Relationship between patient age and $\mathrm{Gl}$ toxicities}

The mean severity of acute GI toxicities was the highest for the 71 to 75 year age-group (4.43) and the lowest for the age group of 76 years or more (1.00). Most patients $(n=37)$ were from this age-group. However, the difference was not statistically significant $(\mathrm{p}=0.238)$.

The incidence of GI toxicities was the highest for the 71 to 75 age-group (45.2\%), followed by the age group of 65 years or less(26.2\%). However, the Chi-Square test proved that the difference in incidence of GI toxicities was not significant between the age groups $(\mathrm{p}=0.265)$. 


\section{Discussion}

The predominant finding from this research study was that neither AP nor lateral patient thickness showed a statistically significant relationship with acute GI toxicities. As such, this study adds to the existing body of knowledge, further clarifying the complex relationship between patient size and toxicities discussed in previously published literature [7-15]. Based on these findings, all patients undergoing PCa radiotherapy may receive similar radiotherapy treatments, without any dosimetric alterations, and similar follow-up care, irrespective of patient size.

The possible cause for this is the use of the VMAT technique which allows to administer more conformal dose to the targets reducing the dose to sensitive OARs such as the rectum. In addition, image-guided radiotherapy is used at a Maltese radiotherapy centre, allowing higher accuracy in the dose administration, with a possible reduction in treatment margins, thereby maintaining a low dose to the OARs $[30,31]$ and limiting GI toxicities, irrespective of patient size.

Patients at the end of treatment showed more GI toxicities. Therefore, patient care should be emphasised in this phase of treatment. A toxicity grading tool (such as the EPIC tools) could be implemented for all patients and treatment sites. This would allow for the incidence and severity of toxicities to be detected and treated accordingly. Moreover, future retrospective analysis of the data could thus be facilitated.

Furthermore, the confounding factors studied did not seem to have a statistically significant effect on toxicities. With regards to the rectal dosimetry, the parameter for all patients in the study was within the recommended tolerance. This would limit the severity of GI toxicities, in line with previous literature [32, 33]. Moreover, only one dose-volume parameter was collected for the rectum (dose to $80 \%$ of the rectal volume). As such, further studies are suggested with more dose-volume parameters for a better understanding of the confounding effect of dose on GI toxicities.

Although the inclusion of pelvic LN in the radiation field would increase the volume of normal tissue being irradiated [2], the tolerance to the rectum was still maintained for patients with LN irradiation. This may explain why no significant increase in the incidence of GI toxicities was observed. In addition, the lack of statistical significance could be due to the fact that the group with LN irradiation had only six patients.

The potential relationship between patient age and toxicities could be due to the physiological changes linked with ageing and co-morbidities. However, this was not established in this study, which was in line with previous literature [34].

Furthermore, PCa radiotherapy treatments may still be improved to further spare normal tissues. The reason being that a significant number of patients experienced a higher incidence of GI toxicities between the first and last week of radiotherapy treatment, since the baseline and follow-up toxicity scores were compared.

\section{Limitations}

Even though the population is small, the whole population was included in the study (exhaustive sampling). Despite the known limitations of retrospective data collection, the EPIC score was systematically used as a data collection tool and very few patients were removed from the sample due to the lack of available data. Moreover, at a Maltese radiotherapy centre, EPIC is filled in by the patient in the presence of a healthcare professional, in order to better explain the questions and avoid misunderstandings. This allows the researchers to confidently argue that patient thickness does not predict well the incidence or the severity of GI side-effects.

Despite the effort to assess the influence of confounding factors, the subgroups for some of these factors were too small to establish valuable conclusions. The influence of LN irradiation (and corresponding larger PTV) on toxicities could not be assessed since only six patients had undergone lymph node irradiation. The same applies to the rectum dose since all patients complied with the dose constraint assessed. A larger sample and more dose-volume parameters are recommended in further studies.

The strength of the study is its novelty. Although the literature includes several studies which examined the association between toxicities and patients' habitus, the conclusions are contradictory. Only one study made use of patient thickness as the measure for patient size, which is directly related to the attenuation of the beam [16]. Moreover, only one similar study utilised the EPIC tool to measure 
toxicities, which is a widespread validated instrument [18]. Therefore, this current study, combining both measurements, provides a novel approach to assessing GI toxicities and patient size. The inclusion of different toxicities, surrogate measurements (such as thickness, weight, BMI), and cancer sites would enrich the study, yet this was not within the scope of this study, which is its limitation. A suggested future study is to compare the relationship between patient size, using different surrogate measurements and assess if they differ in the prediction of different acute and late toxicities (such as GI, urinary, skin, or fatigue).

\section{Conclusions}

In this study, patient AP and lateral thickness were shown to have no significant effect on acute GI toxicities following PCa radiotherapy, when patients are treated with VMAT and image-guided radiotherapy. Therefore, irrespective of their thickness, all patients should be offered the best care and follow-up.

The lack of relationship between patient size and toxicities confirms previously published literature $[9,13,15]$ and contradicts other studies [ 7 , $8,10,11,14]$, showing that toxicities are difficult to predict and may be multifactorial. Since all patients in this study were treated using VMAT and image-guided $\mathrm{CBCT}$, and knowing the advantages of these techniques in terms of conformity, accuracy and decrease in OAR dose, this may explain the lack of difference in GI toxicities for different patient thickness. In addition, since the previous publications used a variety of techniques, this may also influence the results obtained.

However, radiation toxicities remain prevalent, showing that there is still room for improvement of radiotherapy treatments. Lastly, further research is necessary which focuses on different cancers, toxicities, methods of measuring patient size or toxicity grade, and radiotherapy techniques to further elucidate the complex relationship between patient size and radiation toxicities.

\section{Conflict of interest}

None declared.

\section{Funding}

None declared.

\section{Acknowledgements}

The authors wish to express their deepest appreciation to all those who assisted in this research study. In particular, this includes the intermediaries; the statistician who assisted in the data analysis; and those who granted permission for this study to take place.

\section{References}

1. Cherry P, Duxbury AM. Practical radiotherapy physics and equipment. 3rd ed. John Wiley \& Sons, London 2019.

2. Beyzadeoglu M, Ozyigit G, Ebruli C. Radiobiology. In: Basic radiation oncology. 2010th ed. Springer, New York 2010.

3. Peach MS, ShowalterTN, Ohri N. Systematic Review of the Relationship between Acute and Late Gastrointestinal Toxicity after Radiotherapy for Prostate Cancer. Prostate Cancer. 2015; 2015: 624736, doi: 10.1155/2015/624736, indexed in Pubmed: 26697225.

4. World Health Organisation. Cancer country profiles. WHO, Geneva 2014.

5. Flores-Cisneros L, Castro-Eguiluz D, Reyes-Barretero DY, et al. Effects of Dietary Components During and After Concomitant Chemoradiotherapy, Radiotherapy, or Sequential Chemoradiotherapy to the Abdominopelvic Area. Rev Invest Clin. 2018; 70(3): 126-129, doi: 10.24875/ RIC.18002525, indexed in Pubmed: 29943774.

6. Barrett A, Dobbs J, Morris S, Roques T. Practical radiotherapy planning. 4th ed. Hodder Arnold, London 2009.

7. Doi H, Ishimaru F, Tanooka M, et al. Body mass index can affect gastrointestinal and genitourinary toxicity in patients with prostate cancer treated with external beam radiation therapy. Oncol Lett. 2014; 7(1): 209-214, doi: 10.3892/ ol.2013.1658, indexed in Pubmed: 24348850.

8. Meyerhardt JA, Tepper JE, Niedzwiecki D, et al. Impact of body mass index on outcomes and treatment-related toxicity in patients with stage II and III rectal cancer: findings from Intergroup Trial 0114. J Clin Oncol. 2004; 22(4): 648-657, doi: 10.1200/JCO.2004.07.121, indexed in Pubmed: 14966087.

9. Smits A, McGrane J, Lopes A, et al. Radiation-related toxicities and outcomes in endometrial cancer: are obese women at a disadvantage? Int J Clin Oncol. 2017; 22(5): 945-953, doi: 10.1007/s10147-017-1147-5, indexed in Pubmed: 28620815.

10. Doi H, Ishimaru F, Tanooka M, et al. Is obesity correlated with gastrointestinal toxicity induced by radiotherapy for prostate cancer? Radiother Oncol. 2012; 103: S427, doi: 10.1016/s0167-8140(12)71437-8.

11. von Gruenigen VE, Tian C, Frasure $H$, et al. Treatment effects, disease recurrence, and survival in obese women with early endometrial carcinoma : a Gynecologic Oncology Group study. Cancer. 2006; 107(12): 2786-2791, doi: 10.1002/cncr.22351, indexed in Pubmed: 17096437.

12. Kizer NT, Thaker PH, Gao F, et al. The effects of body mass index on complications and survival outcomes in patients with cervical carcinoma undergoing curative chemoradiation therapy. Cancer. 2011; 117(5): 948-956, doi: 10.1002/ cncr.25544, indexed in Pubmed: 20945318.

13. Dandapani SV, Zhang $Y$, Jennelle $R$, et al. RadiationAssociated Toxicities in Obese Women with Endometrial Cancer: More Than Just BMI? ScientificWorldJournal. 2015; 
2015: 483208, doi: $10.1155 / 2015 / 483208$, indexed in Pubmed: 26146653.

14. Smyth H. Does Obesity Impact Treatment Outcome for Prostate Cancer Patients Treated with Radiotherapy: The Weighted Debate. J Cancer Prev Curr Res. 2014; 2(1), doi: 10.15406/jcpcr.2015.02.00021.

15. Showalter TN, Lawrence YR, Xu X, et al. The Influence of Obesity on Toxicity and Biochemical Control after External Beam Radiation Therapy for Prostate Cancer. Int J Radiat Oncol Biol Phys. 2009; 75(3): S351-S352, doi: 10.1016/j. ijrobp.2009.07.806.

16. Wu WC, Chang YR, Lai YL, et al. Impact of body-mass factors on setup displacement during pelvic irradiation in patients with lower abdominal cancer. Radiol Oncol. 2019; 53(2): 256-264, doi: 10.2478/raon-2019-0017, indexed in Pubmed: 30969940.

17. von Hafe $P$, Pina F, Pérez $A$, et al. Visceral fat accumulation as a risk factor for prostate cancer. Obes Res. 2004; 12(12): 1930-1935, doi: 10.1038/oby.2004.242, indexed in Pubmed: 15687393.

18. Koneru H, Cyr R, Feng LiR, et al. The Impact of Obesity on Patient Reported Outcomes Following Stereotactic Body Radiation Therapy for Prostate Cancer. Cureus. 2016; 8(7): e669, doi: 10.7759/cureus.669, indexed in Pubmed: 27551649.

19. Gar-Alnabi MEM, Mohamed EA, Ali Om, et al. Estimation of dose received by sensitive organs in external radiation therapy of cervical carcinoma. Int J Sci Res. 2014; 3(6): 1694-1698.

20. Mitsuyama H, Wallner E, Merrick S. Gregory. Treatment of prostate cancer in obese patients. Oncology. 2006; 20(10): 1191-1197, indexed in Pubmed: 17024870.

21. Brown A, Tan A, Cooper S, et al. Obesity does not influence prostate intrafractional motion. J Med Radiat Sci. 2018; 65(1): 31-38, doi: 10.1002/jmrs.255, indexed in Pubmed: 29359862.

22. Bishop MJ, Miller SR, Joiner MC, et al. Obesity and Hypofractionated Prostate Radiotherapy: Evaluation of Body Mass Index Influence on Dose-Volume Plan Characteristics. Int J Radiat Oncol Biol Phys. 2017; 99(2): E638-E639, doi: 10.1016/j.ijrobp.2017.06.2143.

23. Sabater S, Arenas M, Berenguer R, et al. Body Mass Index and Doses at Organs at Risk in a Mediterranean Population Treated with Postoperative Vaginal Cuff Brachytherapy. Cancer Res Treat. 2015; 47(3): 473-479, doi: 10.4143/ crt.2014.115, indexed in Pubmed: 25672575.

24. Tiberi D, Gruszczynski N, Meissner A, et al. Influence of body mass index and periprostatic fat on rectal dosimetry in permanent seed prostate brachytherapy. Radiat Oncol.
2014; 9(1): 93, doi: 10.1186/1748-717X-9-93, indexed in Pubmed: 24731303.

25. Cesaretti J, Stone N, Stock R. Does prior transurethral resection of prostate compromise brachytherapy quality: A dosimetric analysis. Int J Radiat Oncol Biol Phys. 2004; 60(2): 648-653, doi: 10.1016/s0360-3016(04)00966-6, indexed in Pubmed: 15380602.

26. Michigan Medicine - Urology. Expanded prostate cancer index composite. Updated 2019. https://medicine.umich. edu/dept/urology/research/epic. (11/04, 2019).

27. Wei J, Dunn R, Litwin M, et al. Development and validation of the expanded prostate cancer index composite (EPIC) for comprehensive assessment of health-related quality of life in men with prostate cancer. Urology. 2000; 56(6): 899-905, doi: 10.1016/s0090-4295(00)00858-x, indexed in Pubmed: 11113727.

28. Owens A, Flinton D. Quantitative methods and analysis. In: Ramlau A. ed. Medical imaging and radiotherapy research skills and strategies. 1st ed. Churchill Livingstone, London : 183.

29. Beyzadeoglu M, Ozyigit G, Ebruli C. Genitourinary system cancers. In: Basic radiation oncology 2010th ed. Springer, New york 2010.

30. Deng X, Han Ce, Chen S, et al. Dosimetric benefits of intensity-modulated radiotherapy and volumetricmodulated arc therapy in the treatment of postoperative cervical cancer patients. J Appl Clin Med Phys. 2017; 18(1): 25-31, doi: 10.1002/acm2.12003, indexed in Pubmed: 28291936.

31. Teoh M, Clark CH, Wood K, et al. Volumetric modulated arc therapy: a review of current literature and clinical use in practice. Br J Radiol. 2011;84(1007): 967-996, doi: 10.1259/ bjr/22373346, indexed in Pubmed: 22011829.

32. Barnett GC, West CML, Dunning AM, et al. Normal tissue reactions to radiotherapy: towards tailoring treatment dose by genotype. Nat Rev Cancer. 2009; 9(2): 134-142, doi: 10.1038/nrc2587, indexed in Pubmed: 19148183.

33. Ferreira MR, Thomas K, Truelove L, et al. Dosimetry and Gastrointestinal Toxicity Relationships in a Phase II Trial of Pelvic Lymph Node Radiotherapy in Advanced Localised Prostate Cancer. Clin Oncol (R Coll Radiol). 2019; 31(6): 374-384, doi: 10.1016/j.clon.2019.02.012, indexed in Pubmed: 30902559.

34. Hamstra DA, Stenmark MH, Ritter T, et al. Age and comorbid illness are associated with late rectal toxicity following dose-escalated radiation therapy for prostate cancer. Int J Radiat Oncol Biol Phys. 2013; 85(5): 1246-1253, doi: 10.1016/j.ijrobp.2012.10.042, indexed in Pubmed: 23265567. 